16

\title{
Механизм формирования наноструктур гексагональной симметрии на поверхности металлов последовательностью сдвоенных ультракоротких импульсов излучения ортогональной поляризации
}

\author{
(C) В.С. Макин ${ }^{1,2}$, Ю.И. Пестов ${ }^{3}$, Р.С. Макин ${ }^{4}$ \\ ${ }^{1}$ Филиал АО „Корпорация „Комета“ - „НПЦ ОЭКН“, \\ 194021 Санкт-Петербург, Россия \\ ${ }^{2}$ Санкт-Петербургский политехнический университет - Институт ядерной энергетики, \\ 188540 Сосновый Бор, Ленинградская обл., Россия \\ ${ }^{3}$ АО НИИ оптико-электронного приборостроения, \\ 188540 Сосновый Бор, Ленинградская обл., Россия \\ ${ }^{4}$ Национальный исследовательский ядерный университет „МИФИ“, \\ 115409 Москва, Россия \\ e-mail: makinvs@niioep.ru, ypestov@yandex.ru, makin1946@mail.ru
}

Поступила в редакцию 22.08.2019 г.

В окончательной редакции 21.10.2019 г.

Принята к публикации 28.10.2019 г.

\begin{abstract}
Проанализированы последние экспериментальные результаты по формированию упорядоченных поверхностных наноструктур гексагональной симметрии на металлах под действием серии сканируемых сдвоенных коллинеарных фемтосекундных импульсов ортогональной поляризации с варьируемой временной задержкой. Показано, что образование структур связано с формированием динамической решетки первым импульсом, возбуждением вторым импульсом двух пар поверхностных плазмон-поляритонов в направлениях, симметричных вектору поляризации второго импульса и образующих между собой угол $\pi / 3$, с образованием соответствующих динамических решеток и четырехкратным вырождением первой решетки. Три симметричные относительно нормали к поверхности динамические решетки образуют наблюдаемый экспериментально остаточный поверхностный рельеф гексагональной симметрии.
\end{abstract}

Ключевые слова: поверхностные наноструктуры, динамические решетки, поверхностные плазмон-поляритоны.

DOI: $10.21883 / O S .2020 .02 .48976 .254-19$

В недавних работах по взаимодействию пар импульсов фемтосекундного лазерного излучения с варьируемой временной задержкой с металлами и полупроводниками было обнаружено образование иерархических наноструктур, на ориентацию которых оказывает влияние поляризация излучения $[1,2]$. В недавних работах на металлах было обнаружено образование 2D-структур, обладающих как симметрией второго порядка относительно нормали к поверхности [3], так и гексагональной симметрии [4-6]. Можно предполагать, что на формирование гексагональных структур влияет комплекс параметров, характеризующих сдвоенные импульсы.

В настоящей работе на основе анализа опубликованных экспериментальных данных по образованию 2D-наноструктур на вольфраме [4], нержавеющей стали [5] и кобальте [6] предлагается физическая модель, непротиворечиво объясняющая результат образования двумерных решеток гексагональной симметрии. Такой механизм важен для понимания происходящих физических процессов и дальнейшего развития на его основе одностадийной технологии лазерного наноструктурирования поверхностей конденсированных сред.
В работе [3] при изучении образования нанорельефа поверхности вольфрама под действием серии спаренных импульсов $(v=1 \mathrm{kHz}, \lambda=800 \mathrm{~nm}, \tau=50 \mathrm{fs})$ opтогональной поляризации с фиксированной временной задержкой $\Delta \tau=1.2 \mathrm{ps}$ обнаружено образование почти квадратной двумерной решетки с характерным периодом $d=\lambda / \eta \approx 570 \mathrm{~nm}, \eta \approx 1.4$, в виде цилиндрических выступов на поверхности, где $\eta$ - действительная часть комплексного показателя преломления границы раздела воздух-вольфрам для поверхностных плазмон поляритонов. По двум перпендикулярным направлениям период решетки был одинаков.

В работе [4] в почти аналогичных условиях было обнаружено образование плотно упакованной гексагональной решетки наноструктур, которая когерентно транслировалась при сканировании излучения со скоростью $v=0.06 \mathrm{~mm} / \mathrm{s}$ в направлении вектора напряженности электрического поля $\mathbf{E}_{2}$ второго импульса при плотности энергии в двух импульсах $Q=22.9 \mathrm{~mJ} / \mathrm{cm}^{2}$. Возникает вопрос о физических причинах формирования гексагональных наноструктур. Физика образования квадратной структуры описана в работе [3] и основана на динамическом взаимодействии создаваемой второй ре- 


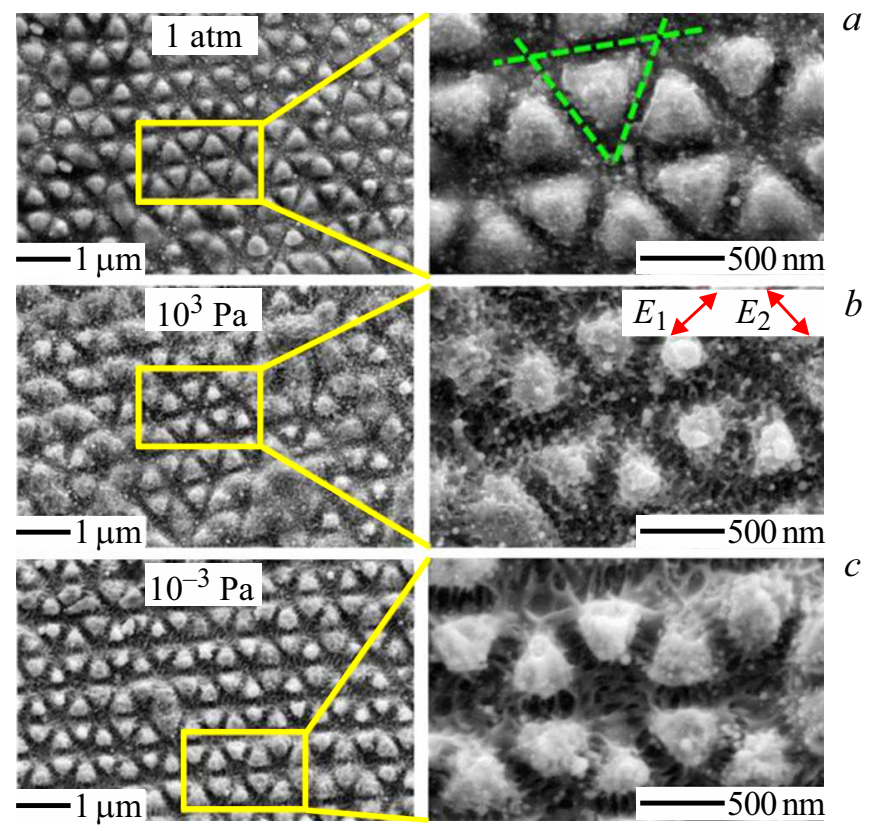

Рис. 1. СЭМ-изображения поверхности двумерных гексагональных структур, полученных путем облучения поверхности вольфрама двумя коллинеарными пучками ортогональной поляризации с временной задержкой; $(a),(b)$ и $(c)$ соответствуют облучению на воздухе при давлениях $1 \mathrm{~atm}, 10^{3} \mathrm{~Pa}$, и $10^{-3} \mathrm{~Pa}$ соответственно [4].

шетки с уже существующей динамической первой. Каждая из динамических решеток формируется в результате интерференции возбуждаемых поверхностных плазмонполяритонов, распространяющихся в ортогональных направлениях, с падающим излучением. Отличие режима образования гексагональных структур от квадратных в значениях реализованных параметров: выше скорость сканирования излучения $(0.06 \mathrm{~mm} / \mathrm{s})$ и ниже плотность энергии в двух импульсах при реализации их равенства в каждом из двух импульсов. Авторы работы [4] предположили, что в процессе формирования гексагональной структуры формируются три решетки с приблизительно равными периодами и ориентациями, отличающимися на угол $\pi / 3$ (рис. 1), однако не указали, за счет чего эти решетки образуются.

Предложим механизм их образования [7]. На рис. 2 приведена круговая векторная диаграмма, иллюстрирующая закон сохранения квазиимпульса при возбуждении поверхностных плазмон-поляритонов (ППП) и поясняющая образование наблюдавшейся в экспериментах с парой задержанных фемтосекундных импульсов ортогональной поляризации гексагональной динамической решетки остаточного поверхностного рельефа. Из диаграммы видно, что первый импульс формирует динамическую решетку с вектором $\mathbf{g}_{1} \| \mathbf{E}_{1}$. Вектор решетки $\mathbf{g}$ определяет ее период $d=2 \pi /|\mathbf{g}|$ и направление $\pm \mathbf{g} /|\mathbf{g}|$. Решетка $\mathbf{g}_{1}$ возникает как результат интерференции падающего излучения и ППП с волновым вектором $+\mathbf{k}_{s 1}$ : $\mathbf{k}_{s 1}=\mathbf{k}_{t}+\mathbf{g}_{1}$, и поскольку $\mathbf{k}_{t}=0$, получаем $\mathbf{g}_{1}=\mathbf{k}_{s 1}$. Здесь $\mathbf{k}_{t}$ - тангенциальная проекция волнового вектора падающего лазерного излучения. Решетка $\mathbf{g}_{1}$ образуется и как результат интерференции с участием ППП с волновым вектором $\left(-\mathbf{k}_{s 1}\right)$ и поэтому оказывается двукратно вырожденной.

Реально в экспериментах всегда образуется некоторый угловой набор (раствор) векторов $\mathbf{g}$ вокруг направления наиболее эффективного возбуждения ППП, задаваемого тангенциальной проекцией вектора напряженности электрического поля лазерного излучения, поскольку возбуждение осуществляется на статистически шероховатой исходной поверхности металла (либо на начальных созданных лазерным излучением локальных разрушениях поверхности „точечного“ типа). При приходе второго импульса для возбуждения ППП в направлении вектора $\mathbf{E}_{2}$ существует исходная статистически шероховатая поверхность, обеспечивающая невысокие амплитуды возбуждаемых ППП. Однако по мере передачи энергии от электронной подсистемы к фононной и формирования динамической решетки рельефа на временах порядка $(1-10)$ ps [8] появляется более эффективный источник возбуждения, связанный с локальными отклонениями резонансных векторов $\mathbf{g}$ от направления $\mathbf{E}_{2}$. Вместе с

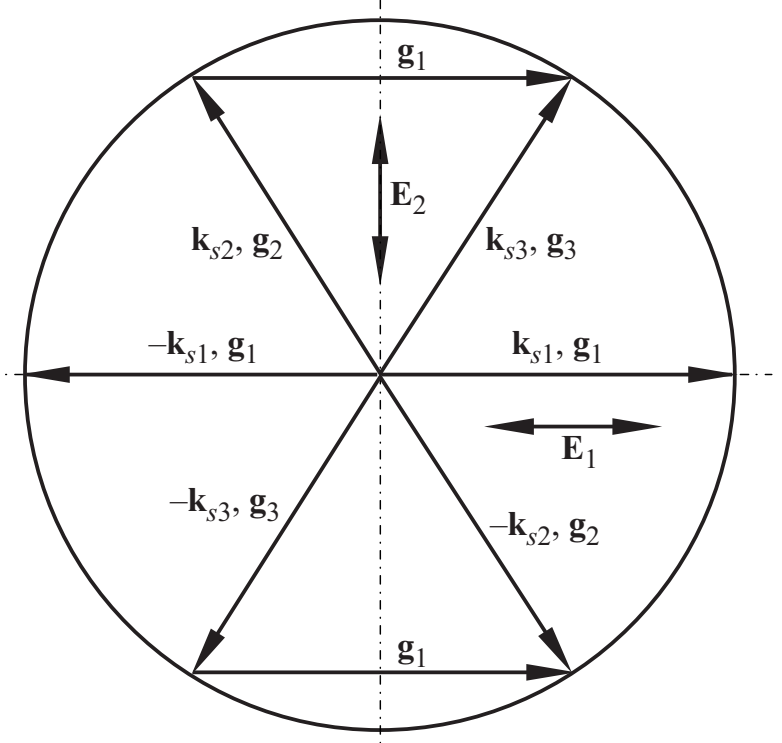

Рис. 2. Круговая векторная диаграмма, иллюстрирующая закон сохранения квазиимпульса и образование динамических решеток при взаимодействии с металлом падающих по нормали к его поверхности последовательности коллинеарных сдвоенных импульсов линейно поляризованного излучения ортогональных поляризаций $\left(\mathbf{E}_{1} \perp \mathbf{E}_{2}\right)$, следующих с задержкой $\Delta \tau \approx(1-10) \mathrm{ps}$, в результате возбуждения ППП с волновыми векторами $\pm \mathbf{k}_{s 1}, \pm \mathbf{k}_{s 2}, \pm \mathbf{k}_{s 3}$ и их интерференции с падающим излучением с образованием периодических структур рельефа с векторами решеток $\mathbf{g}_{1}, \mathbf{g}_{2}, \mathbf{g}_{3}$. Радиус круговой линии равен модулю волнового вектора ППП $\left(\mathbf{k}_{s}\right)$ границы раздела воздух - плазма металла (вольфрама). Решетка $\mathbf{g}_{1}$ оказывается четырежды вырожденной. Остальные обозначения в тексте. 
возникающими переотражениями возбужденных ППП, распространяющихся под углами $\alpha=\left(\mathbf{k}_{s}, \mathbf{E}_{2}\right) \neq 0$ к $\mathbf{E}_{2}$, это приводит к увеличению угла раствора направлений распространения ППП и увеличению их весовых составляющих с $\alpha \neq 0$. С учетом разброса углового набора векторов динамических решеток $\widetilde{\mathbf{g}}_{1}$ оказывается возможной реализация интерференции двух ППП, симметрично распространяющихся под углами, приблизительно равными $\pm 30^{\circ}$ к направлению вектора $\mathbf{E}_{2}$, с падающим излучением (рис. 2) с образованием решеток $\mathbf{g}_{2}$ и $\mathbf{g}_{3}$. Символом „тильда“ здесь обозначен разброс направлений векторов решеток, отклоняющихся от оптимального $\left(\mathbf{g} \| \mathbf{E}_{2}\right)$. Интерференция ППП с волновыми векторами $\mathbf{k}_{s 2}$ и $\mathbf{k}_{s 3}$ вызывает усиление динамической решетки $\mathbf{g}_{1}$. Аналогичная ситуация реализуется для пары ППП с волновыми векторами $\left(-\mathbf{k}_{s 2}\right)$ и $\left(-\mathbf{k}_{s 3}\right)$. Решетка $\mathbf{g}_{1}$ оказывается четырехкратно вырожденной, решетки $\mathbf{g}_{2}$ и $\mathbf{g}_{3}$ - двукратно. Их амплитуды существенно возрастают.

Для вольфрама время задержки между импульсами было фиксированным, $\Delta t=1.2 \mathrm{ps}$ [4] и $\Delta t<\tau_{\mathrm{e}-\mathrm{ph}} \leq$ $\leq 3 \mathrm{ps}$ [9]. Здесь $\tau_{\mathrm{e}-\mathrm{ph}}-$ время электрон-фононной релаксации неравновесных носителей в металле.

Наличие последовательности повторяющихся пар импульсов воздействующего излучения приводит к более четкому выделению трех указанных направлений распространения ППП. Этому способствует также процесс когерентной трансляции рельефа при сканировании лазерного излучения. Углы между сформированными решетками оказываются равными: $\angle\left(\mathbf{g}_{1}, \mathbf{g}_{2}\right)=\pi / 3$ и $\angle\left(\mathbf{g}_{1}, \mathbf{g}_{3}\right)=2 \pi / 3$.

Существование выделенных направлений распространения ППП экспериментально прослеживается и по ориентации решеток высокой пространственной частоты (ВПЧ) в трех симметричных направлениях, формирование которых связано с интерференцией канальных ППП, распространяющихся в противоположных направлениgx [10].

Таким образом, предложенная модель обеспечивает динамическое наложение трех симметрично ориентированных решеток с одинаковыми периодами, которые фиксируются при кристаллизации расплава металла в виде остаточного рельефа. Заметим, что динамический рельеф обычно имеет большую амплитуду по сравнению с остаточным. Масштаб периодических структур, получаемый с участием интерференции ППП выделенных направлений распространения с падающим излучением в режиме взаимодействия динамических решеток, составил $d \approx 710 \mathrm{~nm}<\lambda=800 \mathrm{~nm}$. Исходя из ориентации векторов $\mathbf{E}_{1}$ и $\mathbf{g}_{1}$ на увеличенном изображении результирующих структур (рис. 3) период решетки $d=\lambda / \eta \approx 710 \mathrm{~nm}(\eta \approx 1.12)$. Это означает, что характерная периодичность результирующей структуры по каждому из трех ее направлений несколько меньше длины волны воздействующего лазерного излучения $\lambda$.

Изменение пространственного масштаба сформированных гексагональных структур может быть получено

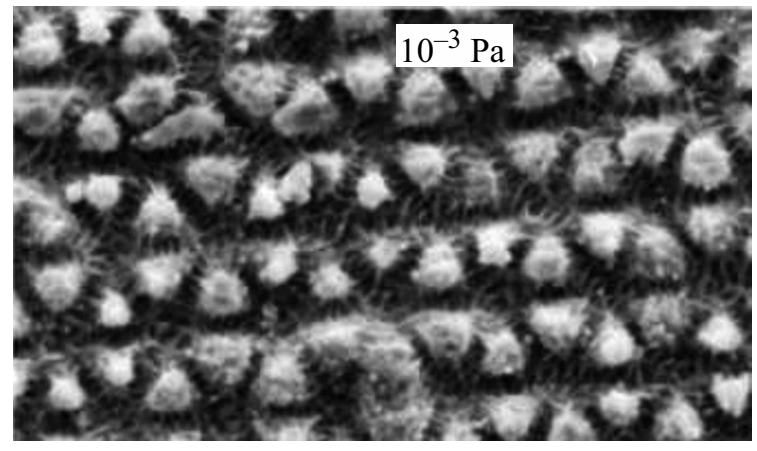

Рис. 3. Увеличенное изображение рис. $1, c$, иллюстрирующее формирование рельефа высокой пространственной частоты на поверхности вольфрама [4].

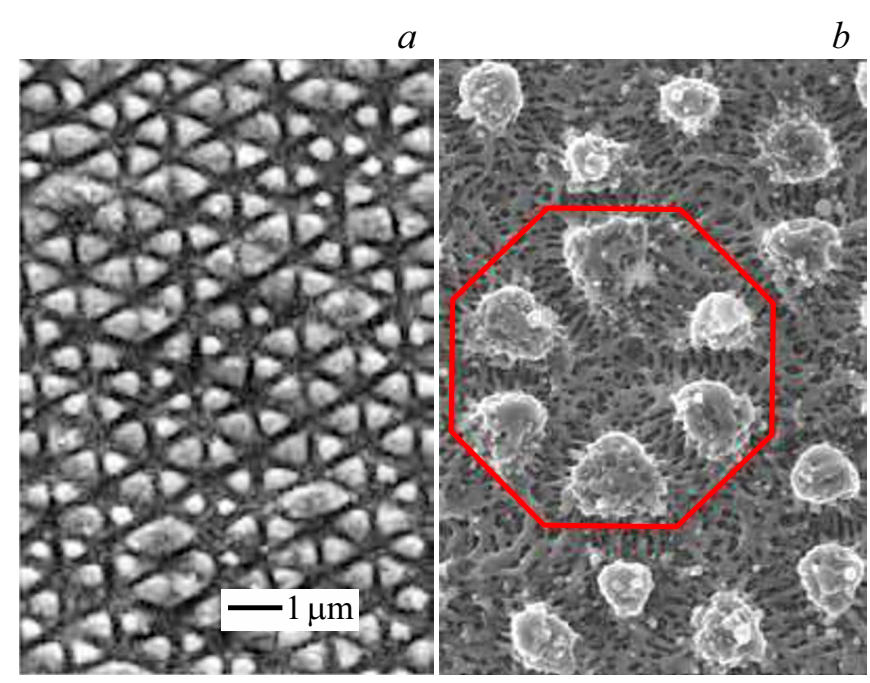

Рис. 4. СЭМ-изображение поверхностей: $(a)$ нержавеющей стали,облученной последовательностью сдвоенных импульсов ортогональной поляризации и задержкой между импульсами $\Delta \tau=5 \mathrm{ps}$, эффективное количество импульсов в пятно равно 10 , расстояние между сканами равно $1 \mu \mathrm{m}$, плотность энергии $Q=0.1 \mathrm{~J} / \mathrm{cm}^{2}[5] ;(b)$ поверхности кобальта при $Q=0.20 \mathrm{~J} / \mathrm{cm}^{2}$ и $\Delta \tau=12 \mathrm{ps}[6]$.

за счет использования другой длины волны воздействующего излучения [5]. Сканирование лазерного излучения, осуществлявшееся в направлении оси симметрии распространения пары возбуждаемых вторым импульсом ППП, при широком фронте распространения структур ( $\approx 4.5 \mathrm{~mm}$, цилиндрическая линза) и оптимальной скорости перемещения пучка обеспечивало когерентную трансляцию гексагональных структур.

При разрушении поверхности кобальта (суммарная плотность энергии в двух импульсах в диапазоне $(0.19-0.23) \mathrm{J} / \mathrm{cm}^{2}$ и задержка $\left.\Delta \tau=12 \mathrm{ps}\right)$ при $Q=0.20 \mathrm{~J} / \mathrm{cm}^{2}$ также наблюдалось образование гексагональных структур с периодом $d \approx 670 \mathrm{~nm}<\lambda$ (рис. 4,b). При больших значениях плотности энергии $Q=0.24 \mathrm{~J} / \mathrm{cm}^{2}$ формировались ромбические структуры. При $Q=0.22 \mathrm{~J} / \mathrm{cm}^{2}$ и $\Delta \tau=24 \mathrm{ps}$ наблюдалось форми- 
рование нанорешеток нормальной ориентации $(\mathbf{g} \| \mathbf{E}) \mathrm{c}$ периодом $d=\lambda / 2 k \eta \approx 95 \mathrm{~nm}, k=4, \eta \approx 1.05$ из обратной универсальности Фейгенбаума [11]. Для объяснения полученных результатов в [6] была предложена модель самоорганизации, основанная на перераспределении локальных областей расплава, обладающих идентичными массами и зарядами в первоначально квадратной решетке в ходе эволюции под действием последующей серии импульсов, в которой использовался метод Эйлера второго порядка.

Подробное описание экспериментальных результатов по формированию гексагональных структур на поверхности нержавеющей стали приводится в [5], однако отсутствует даже предположение о возможном механизме формирования упорядоченного нанорельефа.

Наряду с гексагональными структурами в области облучения в областях понижения основного рельефа [4] при пониженном давлении воздуха $\left(10^{3}, 10^{-3} \mathrm{~Pa}\right)$ хорошо видно формирование решеток ВПЧ $\mathbf{G}$ в промежутке между нанотреугольниками (рис. 3). (Здесь используется другое буквенное обозначение для решеток, образование которых связано с возбуждением и интерференцией групп канальных поверхностных плазмон-поляритонов (КППП) встречных направлений распространения.) Периоды КППП описываются формулой $D=\lambda / 2 k \xi$ при $k=4,8$ и $\xi \geq 1$ [9], где $\xi-$ действительная часть комплексного показателя преломления границы раздела плазма вольфрама-воздух для канальных ППП. Как видно из рис. 3 , направление решеток $\mathbf{G}$ этих структур хорошо коррелирует с направлением векторов $\mathbf{E}_{1}$ и $\mathbf{g}_{2}^{ \pm}$, т.е. с направлениями наиболее эффективного возбуждения КППП. На поверхности кобальта (рис. 4,b) более явно, чем на поверхности вольфрама (рис. 3), видны нанорешетки, сформированные за счет встречной интерференции пространственных гармоник канальных ППП.

Вместе с гексагональными структурами решетки ВПЧ G вносят свой вклад в поглощательную способность наноструктурированной поверхности металла как в процессе воздействия импульсов излучения, так и при измерениях отражательной способности холодной поверхности за счет диссипации энергии возбужденных КППП в металле (ср. с [3]). Сформированная наноструктурированная поверхность вольфрама обладает выраженными поглощательными свойствами „черного“ металла в видимой и ближней ИК спектральных областях, с отражательной способностью $R \leq 0.06$, заметно меньшей, чем при наноструктурировании квадратной решеткой с выступами рельефа в виде нанокружков и характерным пространственным масштабом порядка длины волны лазерного излучения. Существенное увеличение поглощательной способности наноструктурированной поверхности („черный“ металл) [12] с двумя группами нанорешеток, отличающихся пространственными наномасштабами, связано с преобразованием падающего излучения на динамических и остаточных решетках в локализованные и обычные ППП различных направлений распространения с последующей диссипацией их энергии в тепловую энергию.

Когерентная трансляция ячеек поверхностной структуры гексагональной симметрии при сканировании излучения в направлении вектора поляризации второго импульса дала возможность формирования массива элементарных нанотреугольников большой площади. Скорость формирования нанотреугольников в реализованных экспериментальных условиях составила $\sim 1.5 \cdot 10^{5} \mathrm{~s}^{-1}[4]$.

Формирование гексагонального рельефа поверхности носит достаточно общий характер, поскольку было экспериментально обнаружено на различных металлах: вольфраме (рис. 1), нержавеющей стали (рис. 4,a) и кобальте (рис. $4, b)$. Создание оптимальных условий для формирования массива гексагонального рельефа в виде острийных структур позволило бы получить воспроизводимую одностадийную технологию формирования поверхности „черного“ металла [12] и заметно увеличить поглощательную и эмиссионную способности поверхности, создать поверхность для полевой эмиссии электронов. Заметим, что обнаружены режимы формирования гексагональных структур излучением круговой поляризации как серией единичных импульсов $[13,14]$, так и сдвоенных с противоположным направлением вращения [5]. Они также хорошо объясняются в рамках универсальной поляритонной модели [11].

Таким образом, предложен физический механизм образования наноструктур гексагональной симметрии при нелинейном взаимодействии серии сдвоенных коллинеарных импульсов ортогональной поляризации с изменяемой временной задержкой с поверхностью металла. Механизм основан на динамическом возбуждении двух пар ППП, распространяющихся в симметричных относительно вектора поляризации второго импульса направлениях и усиливающих динамическую решетку, созданную первым импульсом. Пары ППП противоположных направлений распространения формируют две решетки при интерференции с падающей волной. В результате в динамике образуются три решетки, определяющие гексагональную симметрию облученной поверхности. Остаточные симметричные решетки двух различных пространственных наномасштабов обеспечивают высокую поглощательную способность облученной поверхности в видимой и ближней ИК областях спектра. Предложенный подход позволит оптимизировать процесс получения больших массивов гексагонально наноструктурированных поверхностей „черных“ металлов для различных металлов.

\section{Благодарности}

Авторы выражают благодарность профессору Чунле Гуо за полезные обсуждения материалов статьи, а также И.В. Аркановой за подготовку рисунков. 


\section{Конфликт интересов}

Авторы завляют, что у них нет конфликта интересов.

\section{Список литературы}

[1] Bonse J., Hohm S., Kirner S.V., Rosenfeld A., Kruger J. // IEEE J. Selected Topics. 2017. V. 23. N 3. P. 9000516.

[2] Vorobyev A.Y., Guo C. // Laser Photonics Rev. 2013. V. 7. N 3. P. 385.

[3] Qiao J., Yang F., Wang Y., Yang J., Sun. // Optics Express. 2015. V. 23. N 20. P. 26617.

[4] Liu Q., Zhang N., Yang J., Qiao H., Guo C. // Optics Express. 2018. V. 26. N 9. P. 11718.

[5] Fraggelakis F., Mincuzzi G., Lopez J., Manek-Honninger I., Kling R. // Appl. Surf. Sci. 2019. V. 470. P. 677.

[6] Jalil S.A., Yang J., ElKabbash M., Cong C., Guo C. // Appl. Phys. Lett. 2019. V. 115. P. 031601.

[7] Бажсенов В.В., Бонч-Бруевич А.М., Либенсон М.Н., Макин В.С. // Письма в ЖТФ. 1984. Т. 10. В. 24. С. 1520.

[8] Zhigilei L.V., Lin Z., Ivanov D.S. // J. Phys. Chem. C. 2009. V. 113. P. 11892

[9] Агранат М.Б., Ашитков С.И., Овчинников А.В., Ситников Д.С., Юркевич А.А., Чеббонов О.В., Перельман Л.Т., Анисимов С.И., Фортов В.Е. // Письма в ЖЭТФ. 2015. T. 101. B. 9. C. $671-676$.

[10] Макин В.С., Логачева Е.И., Макин Р.С. // Опт. и спектр. 2016. T. 120. № 4. С. 118.

[11] Макин В.С., Макин Р.С., Воробьев А.Я., Гуо С. // Нелинейность в современном естествознании / Под ред. Малинецкого Г.Г. Изд-во ЛКИ, 2009. С. 303.

[12] Vorobyev A.Y., Makin V.S., Guo C. // Phys. Rev. Lett. 2009. V. 102. P. 234301.

[13] He Y., Zhang J., Singh S., Garcell E.1., Vorobyev A.Y., Lam B., Zhan Z., Yang J., Guo C. // Appl. Phys. Lett. 2019. V. 114. P. 133101

[14] Romano J.M., Garcia-Giron A., Penchev P., Dimov S.S.S. // Appl. Surf. Sci. 2018. V. 440. P. 162-169. 